\title{
Outcomes of fifty patients entering an adolescent bariatric surgery programme
}

Billy White ${ }^{1,2}$

Jackie Doyle ${ }^{3}$

Kirsten Matschull 2

Marco Adamo ${ }^{4}$

Deborah Christie ${ }^{2}$

Dasha Nicholls ${ }^{5}$

Sanjay Kinra ${ }^{6}$

Ian CK Wong ${ }^{7}$

Russell M Viner ${ }^{1,2}$

1 Department of Population Health Sciences, UCL Institute of Child Health, London, UK

${ }^{2}$ Department of Adolescent Medicine, University College London Hospital, London, UK

${ }^{3}$ Department of Child and Adolescent Psychological Services, University College London Hospital, London, UK

${ }^{4}$ Department of Surgery, University College London Hospital, London, UK

${ }^{5}$ Feeding and Eating Disorders Unit, Great Ormond Street Hospital, London, UK

${ }^{6}$ Department of Non-communicable disease epidemiology, London School of Hygiene and Tropical Medicine, London, UK

${ }^{7}$ Centre for Paediatric Pharmacy Research, UCL School of Pharmacy, United Kingdom 
Corresponding Author: Dr Billy White, 250 Euston Road, London NW1 2PG, UK. Tel: 02034479489

Email: billy.white@ucl.ac.uk

Keywords: bariatric surgery, obesity, adolescent, child

Word count: 2408

\section{Abstract}

Objective. Bariatric surgery is the most effective intervention for weight loss and obesity-related comorbidities currently available. Little is known about adolescents entering NHS bariatric programmes. We aimed to characterize those entering a pathway, and report their outcomes.

Design. Prospective service evaluation of patients assessed within a single NHS adolescent bariatric service.

Results. 50 patients assessed between 26th July 2007 and 27th January 2014; 6 (12\%) were not eligible for surgery, $7(14 \%)$ actively opted out, $8(16 \%)$ were lost to follow-up and 29(58\%) underwent surgery (18 sleeve gastrectomy (SG) 11 Roux-en-y gastric bypass (RYGB) and 0 adjustable gastric band). Mean (SD) age at initial assessment was 16.0(1.3) years and 18.3(1.3) at surgery (youngest 15.7 years). Mean time taken to surgery was 1.8 years; longer in those with higher BMI and aged below 14 at first assessment. Mean (SD) BMl at surgery was 53.1 (8.3) kg/m², lower in those undergoing RYGB $(-5.2,95 \% \mathrm{Cl}-11.6,1.13)$. Follow-up was inconsistent and challenging; $1 / 29$ (3.5\%) was transferred to a regional centre, 10/29 (34.5\%) attended ongoing follow-up within our protocol, 6/29 (20.7\%) had intermittent monitoring and 12/29 (41.4\%) were lost to follow-up. Mean BMI change at 1 year $\left(-14.0 \mathrm{~kg} / \mathrm{m}^{2}\right)$ and complications were similar to published cohorts. Data from 11 lost to follow-up were obtained and outcomes appeared similar to those actively followed-up.

Conclusion. Adolescent bariatric surgery in the NHS appears effective, with outcomes similar to those 
reported internationally. Further work is needed to optimise post-surgical surveillance, and reduce age at surgery.

\section{Introduction}

Bariatric surgery is the only intervention resulting in clinically meaningful weight loss for obese children and young people.(1) In the UK, national guidelines for adolescent bariatric surgery are contained within the most recent NICE obesity guideline(2).

The largest and most complete bariatric research cohorts come from the US $(3,4)$ and Sweden(5) comprising between 25 and 242 adolescents undergoing surgery, compared with very small case reports totaling 20 adolescents in the UK.(6-8) A recent systematic review of adolescent bariatric surgery showed mean loss of $13.5 \mathrm{~kg} / \mathrm{m}^{2}$ at 1 year post-surgery with greatest change seen in those undergoing Roux-en-Y gastric bypass (RYGB), followed by sleeve gastrectomy (SG) and adjustable gastric band (AGB).(1) Such changes are very much greater than those from with drug treatments such as metformin (mean loss $\left.1.4 \mathrm{~kg} / \mathrm{m}^{2}\right)(9)$, orlistat $(-0.8$ $\left.\mathrm{kg} / \mathrm{m}^{2}\right)(10)$ or lifestyle interventions $\left(-1.25 \mathrm{~kg} / \mathrm{m}^{2}\right) .(11)$

Outcome studies have mostly focused on those who received surgery,(1) with only one service in Saudi Arabia publishing pathway outcomes in a clinical service.(12) It is unclear how many young people who are referred for surgery actually receive it, and the reasons for not receiving it in those referred. Furthermore, the outcomes of those entering a clinical programme compared to a research cohort are largely unknown. Such data are important for planning and evaluating adolescent surgery programmes, particularly within statefunded systems.

Our aim was to characterize the patients referred to an adolescent bariatric surgery programme within the NHS in England and report their outcomes, including number reaching surgery, change in BMI and complications. We present data for the first 50 patients assessed within a single service.

\section{Methods}

We reviewed data on prospectively-identified adolescent patients referred to University College London Hospitals (UCLH) for consideration of bariatric surgery. Inclusion criteria were age less than 18 years and first assessed between $1^{\text {st }}$ July 2007 and 27 th January 2014. 
Body weight was measured using Tanita BC-418MA or SECA 645 scales, height using a wall-mounted stadiometer. BMI was calculated as weight $/$ height $^{2}\left(\mathrm{~kg} / \mathrm{m}^{2}\right)$ and $\mathrm{zBMI}$ using UK reference data. (13)

We screened for diabetes using $\mathrm{HbA} 1 \mathrm{c}$ and fasting glucose; hypertension using automated blood pressure monitor (Datascope Accutor Plus)(14) with appropriately sized cuff and UK thresholds(15); and raised lipids using American Heart Association thresholds. (16) Those having surgery had a standard oral glucose tolerance tests (OGTT) (17) and where clinically indicated, screening for sleep apnoea and polycystic ovarian syndrome. (18) Previous screening results were used in those who left pre-surgery and refused screening. All participants were invited to participate in the Genetics of Obesity Study to enable identification of monogenic causes of obesity.(19)

Socio-economic status was derived from the patient's home postcode using the 2007 Index of Multiple Deprivation (IMD, England only), and grouped into five deprivation quintiles (lowest quintile defined as "deprived").(20) Ethnicity was self-reported using a list used by the hospital.

We assumed lack of interest if two successive pre-operative appointments were missed and we were unable to contact families by telephone or letter. All participants were reviewed by a clinical psychologist to assess Gillick competency and screen for mental health co-morbidities. Eligible patients were offered choice of RYGB or SG procedure once weight gain had ceased, with those having higher BMI encouraged to undergo SG (surgeon preference due to safety concerns). We used patient records to collect BMI trajectories and comorbidity data. We attempted to contact all post-operative patients who had not attended follow-up within the previous 6 months if they were in the first two post-operative years, or 1 year after that. Where we were unable to contact participants, we gathered information from their parent or GP (self-reported BMI not included in trajectory analyses).

Analyses. We used descriptive statistics to describe patients flow, student-t and chi ${ }^{2}$ tests to compare group differences and regression models to analyse time taken to surgery. Data nearest 1-year post-surgery were used to calculate BMI change at 1 year (8-16 months only).

Study approval. This study fulfilled National Research Ethics Service (NRES) criteria for service evaluation, and was registered in the UCLH Research \& Development Department. 


\section{Results}

Pre-operative patient flow. Figure 1 summarises patient flow. A total of 50 patients were assessed; 7(14\%) actively withdrew from the pre-operative pathway, 8 (16\%) did not attend to complete assessments, $6(12 \%)$ were deemed ineligible and 29 (58\%) underwent bariatric surgery.

Reasons given for active withdrawal were the wish to pursue non-surgical interventions and stress of coming into a London hospital; none were due to lack of funding. Principal reasons for exclusion were: residence outside the UK making post-operative monitoring unsafe $(n=1)$, inability to consent due to learning difficulties $(n=2)$, severe needle phobia $(n=1)$, inconsistent desire to undergo bariatric surgery $(n=1)$, and complex behavioural difficulties $(n=1)$. None were excluded because of psychiatric disorders. One deemed ineligible subsequently underwent surgery in the private sector.

Patient characteristics. Table 1 summarises demographics and co-morbidities. Distance from patient home to UCLH were $<10$ miles in 20/50 (30\%), $<50$ miles in $39 / 50$ (78\%) and $<100$ miles in $46 / 50(92 \%)$. Three had hypothalamic obesity secondary to previous treatment of hypothalamic-pituitary tumours; the two with previous craniopharyngioma underwent SG but given their outcome (described below), surgery was not offered to the subsequent patient with optic glioma. One with an identified obesity-promoting monogenic genetic variant (homozygous leptin receptor mutation) with one with achondroplasia underwent surgery.

Access to surgery. There was no difference in gender, BMI or age between those having surgery, and those not $(p<0.05)$. Deprivation data were available for 48 participants ( 1 from Scotland, 1 postcode not registered on IMD). Graphically it appears that patients from more deprived populations were less likely to get to surgery (Figure 2), however there was no difference in access to surgery between those in the most deprived quintile and the remainder of the cohort.

Mean time from assessment to surgery was 1.8 years (range $0.5-4$ years). All 4 patients entering the pathway below 14 years of age waited at least 2.5 years for surgery. There was moderate evidence that baseline BMI $\left(0.04\right.$ years per $\left.\mathrm{kg} / \mathrm{m}^{2}(95 \% \mathrm{Cl}-0.00,0.08)\right)$ but not duration of program $(-0.08$ per year ($0.30,0.13))$ or age $(0.10$ per year $(-0.34,0.55))$ was associated with increased time to surgery in those aged $>=14$ years at programme entry. Temporary cessation of the programme for 6 months in 2013 due to reorganization of the service resulted in delays at that time. 
Surgery details. A total of 18 patients underwent SG and 11 RYGB. One RYGB procedure included use of a Fobi ring. All procedures were performed by two successive bariatric surgeons fulfilling British Obesity \& Metabolic Surgery Society (BOMSS) competencies, and reporting outcomes to National Bariatric Surgery Registry (NBSR). Participants fulfilled NICE criteria including BMI greater than $40 \mathrm{~kg} / \mathrm{m}^{2}$ at the time of surgery planning. (2)

Mean (SD) BMI was 49.8 (7.0) kg/m² for those undergoing RYGB, and 55.0 (8.7) for SG. There was moderate evidence that those undergoing RYGB had lower BMI (difference in means $-5.2,95 \% \mathrm{Cl}-11.5$, 1.1). Mean (SD) age at surgery was $18.3(1.3)$ years with no difference between procedures $(-0.6,95 \% \mathrm{Cl}$ $1.5,0.4)$. The youngest was aged 15.7 years; two were aged under 16 years, eight were $16-17$ years and the remaining nineteen were 18 years or over.

Weight outcomes. Follow-up post-surgery was inconsistent. At the time of analysis, patients were mean 4.0 years (SD 1.9) post-surgery (range 1.4-7.9). All were offered follow-up at our centre. One was transferred to a regional centre for ongoing follow-up. Of the remaining, 26.3\% (10/28) attended ongoing follow-up within our protocol, $21.4 \%$ (6/28) had intermittent monitoring, 42.9\%(12/28) were lost to follow-up and. Follow-up was achieved in $79.3 \%$ at 1 -year post-surgery (23 of the 29 reaching 1 year since surgery), $59.1 \%$ at 2 years (13 of 22$)$ and $33.3 \%$ at 5 years (3 of 9$)$.

BMI data at 1-year were available for 23 patients (11 RYGB, 12 SG), with no evidence of difference in means between the two procedures (mean (SD) RYGB -14.8 (5.5), SG -13.4(10.8), p=0.7). Greater variation in BMI change at 1 year was seen in patients undergoing SG than RYGB.

Figure 3 shows the BMI trajectory of each patient relative to BMI at surgery. A substantial variability in BMI trajectories was seen across subjects. Data were predominantly limited to 3 years after surgery. In line with published studies, the majority of patients lost weight rapidly in the first 6 months $\left(10-20 \mathrm{~kg} / \mathrm{m}^{2}\right)$, with subsequent deceleration in the rate of weight loss in the next 6 months and weight stability in the second year.

The patient with achondroplasia lost over $30 \mathrm{~kg} / \mathrm{m}^{2}$ in the first year after surgery; however, his stature makes BMI hard to interpret. Of the two patients with previous craniopharyngioma (both underwent SG), one regained all BMI loss and the other lost $23.2 \mathrm{~kg} / \mathrm{m} 2$ after 6.3 years follow-up. Two further patients regained all weight loss; this included one with leptin receptor variant whose genetic variant was not expected to respond 
poorly to surgery (personal communication with Prof Sadaf Farooqi, University of Cambridge).

Outcome data were gained from 16 of 17 participants with intermittent or inactive follow-up ( 8 from young person, 4 from parents and 4 from GPs); the remaining patient had no identifiable contact details or GP. None had ongoing local specialist monitoring for complications or micronutrient status. They reported BMI trajectories in the range of those attending follow-up (one GP was not aware of their patient's weight trajectory); one reported regain of all post-operative weight loss and one mother reported an unspecified amount of weight regain after removal of the Fobi ring; neither had any identified co-morbidities suggesting they may respond poorly to surgery.

Co-morbidities and complications. No patients died post-operatively; 2/29 (6.9\%) patients had a gastric perforation, one identified in the immediate post-operative period after RYGB and the second 1-year after surgery. Both were successfully repaired by laparoscopy with no further sequelae. 2/29 (6.9\%) patients had a gastro-jejunal stricture, both treated after respectively one and two endoscopic dilatations.

The patient with previous craniopharngioma and subsequent cerebral vascular disease and cerebral vascular events (CVE) had a further CVE in the peri-operative period resulting in hemiplegia, expressive and receptive dysphasia and loss of thirst. He required prolonged neuro-rehabilitation with residual loss of function. The following sequelae were also reported: gall stones requiring cholecystectomy $(n=3)$, excess skin (10), pregnancy (2), gastro-eosophageal reflux (7), unexplained abdominal pain (2), constipation (1), oesophagitis resolved after removal of Fobi ring (1), hypotension (1 postural, 1 cause unknown), ulcerative colitis with biliary cirrhosis and gastroparesis (1), poorly controlled PCOS (2), skin infections (1) and dumping syndrome (1).

Data were insufficient to allow detailed analysis of resolution of co-morbidities or cardio-metabolic risk factors post-operatively, with the exception of diabetes. Diabetes control improved in all three patients with diabetes; two were able to stop all hypoglycaemic agents.

\section{Discussion}

This is the first study describing the characteristics and outcomes of patients entering an adolescent bariatric surgical programme in the NHS. We found that over half of adolescents seen were both eligible and interested in surgery. Main reasons for non-eligibility were lack of capacity to consent, behavioural difficulties 
and inconsistent desire for surgery. Patients had relatively few identified co-morbidities and surgery was undertaken mainly for weight loss rather than control of obesity-related conditions.

Change in BMI, mortality and complications were within the range described in systematic reviews. $(1,21)$ Patients undergoing SG had a higher baseline BMI and greater variation in BMI change after surgery compared to those having a RYGB. High rates of attrition to follow-up were seen after surgery, despite earlier careful identification and support of medical and psychological co-morbidities, and good adherence to the pre-operative preparation programme.

These data raise important issues. First, we were referred low numbers of adolescents with weight-related co-morbidities that respond well to bariatric surgery, such as type 2 diabetes, sleep apnoea and idiopathic intracranial hypertension.(22-24) We hope that raised awareness of the safety and efficacy of bariatric surgery to control these conditions will increase referrals. Subsequent to this cohort, we have seen an increase in patient referrals, particularly those with type 2 diabetes.

Second, the mean age at surgery was 18 years of age and the youngest 15.7 years. This compares to a mean age of 16.5 and 17.1 years in larger cohorts described previously. $(4,5)$ This may reflect time taken from first assessment to surgery, or reluctance to refer younger patients in the NHS. NICE guidance recommends surgery in those who have "nearly reached physiological maturity", a conservative recommendation compared to other international guidelines.(25) Earlier referrals may help reduce the age at surgery, and change the BMI trajectory of this population.

Third, we encountered various dilemmas about eligibility for surgery, particularly for those who perceived that surgery was the only remaining treatment option. A systematic review undertaken by these authors has shown that there is a limited evidence base on psychosocial predictors of outcome after surgery in adolescence, with only two studies comparing baseline psychosocial variables and BMI outcomes. (26) In addition, we reviewed three patients with previous pituitary-hypothalmic tumours. Two underwent a bariatric procedure (SG) with disappointing results. A subsequently published small case series suggest improved outcomes after RYGB compared to SG in those with hypothalamic obesity,(27) however small numbers and non-randomised methodology limit generalizability.

Fourth, there was significant attrition both before and after surgery. Our data suggest a non-significant trend for those with lower SES to be more likely to drop out pre-surgery, and it will be important to monitor and 
facilitate access of deprived groups to surgery. Our post-operative attrition was high compared to research cohorts who achieved follow-up rates of $89-100 \%$ at 2 years through telephone surveillance and home visits. $(4,5,28)$ Those not attending follow-up largely reported doing equally well as those attending follow-up, and attrition was largely due to the practicalities of attending follow-up rather than poor outcomes. Attrition has subsequently improved with the appointment of a dedicated adolescent specialist nurse who provided email, SMS and telephone monitoring in addition to standard clinic appointments.

Lastly, patients frequently described issues with excess skin, with difficulty accessing plastic surgery in the NHS due to variations in funding and eligibility criteria.

Limitations. Our study is subject to the common limitations of service evaluation studies; e.g. data were not collected at standardised timepoints and collection of co-morbidity data at follow-up was also not standardized, limiting data availability. We did not routinely collect data on quality of life or psychosocial functioning. We minimized bias due to attrition by contacting those not attending follow-up. Self-reported weight trajectories were similar to that of those attending follow-up, and their complications have been included in this report. The majority of those who decided against surgery did noas tht remain within our service, and we are unable to compare their outcomes to those who had surgery.

\section{Conclusions}

Adolescent bariatric surgery in an NHS service compares favourably to international cohorts, and shows promise as an effective treatment for severe obesity. Further work is needed to improve patient selection, reduce age at surgery and attrition.

\section{Statements}

Conflict of interest. The authors declared no conflict of interest. This article presents independent research funded by the National Institute for Health Research (NIHR) in England under its Programme Grants for Applied Research programme (RP-PG-0608-10035). The views expressed in this publication are those of the authors and do not necessarily reflect those of the NHS, the NIHR, or the Department of Health.

Endnote. Subsequent to this study period, a total of 36 young people initially assessed in the adolescent pathway have undergone bariatric surgery at UCLH. 
Contributorship statement. BW collected and analysed the data, drafted and revised the paper. He is guarantor. KM was involved in data collection. JD, DC, DN, MA, SK, and IW were all involved in the conception of the study, and revised the draft paper. RV initiated the concept, supervised data analysis, and drafted and revised the paper. All approved the final version of the paper.

Licence statement. The Corresponding Author has the right to grant on behalf of all authors and does grant on behalf of all authors, an exclusive licence (or non-exclusive for government employees) on a worldwide basis to the BMJ and co-owners or contracting owning societies (where published by the BMJ on their behalf), and its Licensees to permit this article (if accepted) to be published in Archives of Disease in Childhood and any other BMJ products and to exploit all subsidiary rights, as set out in our licence.

\section{"What is already known on this topic"}

- Bariatric surgery in adolescence at one year has comparable outcomes as adults, with greatest BMI change seen in those undergoing Roux-en-Y gastric bypass

- Bariatric surgery results in greater BMI change compared to lifestyle and drug interventions

- Increasing numbers of bariatric procedures in adolescents are undertaken worldwide each year, however NICE recommends surgery only in exceptional circumstances in adolescents.

\section{"What this study adds"}

- BMI outcomes and complications in those undergoing surgery in a clinical NHS bariatric service are equivalent to international cohorts, with similar complication rates and no deaths.

- Adolescents undergoing surgery in an NHS adolescent bariatric pathway were older than those in published cohorts, with longer time taken to reach surgery.

- Attrition was common and higher than seen in research cohorts. There were suggestions that low SES influenced access to surgery. Those not attending follow-up appeared to have similar outcomes, but did not have micronutrient surveillance. Home visits and telephone surveillance appears useful in research cohorts and should be considered in clinical services.

\section{References:}


1. Black J, White B, Viner R, et al. Bariatric surgery for obese children and adolescents: a systematic review and meta-analysis. Obes Rev 2013;14:634-44.

2. National Institute for Health and Care Excellence. Obesity: identification, assessment and management of overweight and obesity in children, young people and adults. National Insitute for Health and Clinical Excellence, 2014.

3. Sysko R, Zakarin EB, Devlin MJ, et al. A latent class analysis of psychiatric symptoms among 125 adolescents in a bariatric surgery program. International Journal of Pediatric Obesity 2011;6:289-97.

4. Inge TH, Courcoulas AP, Jenkins TM, et al. Weight Loss and Health Status 3 Years after Bariatric Surgery in Adolescents. N Engl J Med 2016;374:113-23.

5. Olbers T, Gronowitz E, Werling M, et al. Two-year outcome of laparoscopic Roux-en-Y gastric bypass in adolescents with severe obesity: Results from a Swedish Nationwide Study (AMOS). International Journal of Obesity 2012;36:1388-95.

6. Sachdev P, Makaya T, Marven S, et al. Bariatric surgery in severely obese adolescents a single centre experience. Archives of Disease in Childhood 2012;97:A103-A4.

7. White B, Finer N, Adamo M, et al. Outcomes of an adolescent bariatric service. Archives of Disease in Childhood 2012;97:A63.

8. Ford-Adams M, Mortimer H, Bevan D, et al. G98 Multidisciplinary Assessment For Bariatric Surgery in Adolescents: A Pilot Project from a National Referral Service. Archives of Disease in Childhood 2013;98:A47-A8.

9. Park MH, Kinra S, Ward KJ, et al. Metformin for obesity in children and adolescents: a systematic review. Diabetes Care 2009;32:1743-5.

10. Viner RM, Hsia Y, Tomsic T, et al. Efficacy and safety of anti-obesity drugs in children and adolescents: systematic review and meta-analysis. Obesity reviews 2010;11:593-602.

11. Ho M, Garnett SP, Baur LA, et al. Effectiveness of Lifestyle Interventions in Child Obesity: Systematic Review With Meta-analysis. Pediatrics 2012;130:e1647.

12. Alqahtani AR, Elahmedi MO. Pediatric Bariatric Surgery: The Clinical Pathway. Obesity surgery 2015;25:910-21.

13. Cole TJ, Freeman JV, Preece MA. Body mass index reference curves for the UK, 1990. Arch Dis Child 1995;73:25-9.

14. Wong S-N, Tz Sung RY, Leung LC-K. Validation of three oscillometric blood pressure devices against auscultatory mercury sphygmomanometer in children. Blood Pressure Monitoring 2006;11:281-91.

15. Jackson LV, Thalange NKS, Cole TJ. Blood pressure centiles for Great Britain. Archives of Disease in Childhood 2007;92:298-303.

16. NCEP Expert Panel on Blood Cholesterol Levels in Children and Adolescents. National Cholesterol Education Program (NCEP): Highlights of the Report of the Expert Panel on Blood 
Cholesterol Levels in Children and Adolescents. Pediatrics 1992;89:495-501.

17. American Diabetes Association. Standards of Medical Care in Diabetes Diabetes Care 2010;33:S11-S61.

18. Viner RM, White B, Barrett T, et al. Assessment of childhood obesity in secondary care: OSCA consensus statement. Archives of disease in childhood Education and practice edition 2012;97:98-105.

19. Genetics of obesity study [cited 28th April 2017]. Available from: http://www.goos.org.uk/.

20. Department for Communities and Local Government. English Indices of Deprivation 20102010 [8th August 2015]. Available from: data.gov.uk/dataset/index-of-multipledeprivation.

21. Paulus GF, de Vaan LEG, Verdam FJ, et al. Bariatric Surgery in Morbidly Obese Adolescents: a Systematic Review and Meta-analysis. Obesity surgery 2015;25:860-78.

22. Sjöström L. Review of the key results from the Swedish Obese Subjects (SOS) trial - a prospective controlled intervention study of bariatric surgery. Journal of Internal Medicine 2013;273:219-34.

23. Haines KL, Nelson LG, Gonzalez R, et al. Objective evidence that bariatric surgery improves obesity-related obstructive sleep apnea. Surgery 141:354-8.

24. Handley JD, Baruah BP, Williams DM, et al. Bariatric surgery as a treatment for idiopathic intracranial hypertension: a systematic review. Surgery for Obesity and Related Diseases 11:1396-403.

25. Aikenhead A, Lobstein T, Knai C. Review of current guidelines on adolescent bariatric surgery. Clinical Obesity 2011;1:3-11.

26. White B, Doyle J, Colville S, et al. Systematic review of psychological and social outcomes of adolescents undergoing bariatric surgery, and predictors of success. Clinical Obesity 2015;5:312-24.

27. Weismann D, Pelka T, Bender G, et al. Bariatric surgery for morbid obesity in craniopharyngioma. Clinical endocrinology 2012.

28. O'Brien PE, Sawyer SM, Laurie C, et al. Laparoscopic Adjustable Gastric Banding in Severely Obese Adolescents. A randomised trial. JAMA 2010;303:519-26. 


\section{Tables}

Whole cohort

No surgery

Surgery

p

Number

Female $\mathrm{n}(\%)$

Mean (SD) age at baseline

Age range at baseline

Mean (SD) baseline BMI $\left(\mathrm{kg} / \mathrm{m}^{2}\right)$

Mean baseline zBMI

BMl range $\left(\mathrm{kg} / \mathrm{m}^{2}\right)$
50

$29(58.0)$

$16.0(1.3)$

$12.8-18.5$

$51.2(7.5)$

$4.21(0.34)$

$37.5-69.6$
21

$11(52.4) \quad 18(62.1)$

$15.9(1.3)$

$16.1(1.3)$

$12.8-17.8$

13.4-18.5

0.6

$51.1(7.4) \quad 51.3(7.8) \quad 0.9$

$4.21(0.3) \quad 4.20(0.3) \quad 1.0$

$37.5-64.8 \quad 38.2-69.6$ 


$\begin{array}{lllll}\text { British } & 29(58.0 \%) & 14(66.7 \%) & 15(51.7 \%) & \\ \text { African/Caribbean } & 8(16.0 \%) & 3(14.3 \%) & 5(17.3 \%) & 0.7 \\ \text { Asian } & 5(10.0 \%) & 1(4.8 \%) & 4(13.8 \%) & \\ \text { Other } & 8(16.0 \%) & 2(14.3 \%) & 5(17.2 \%) & \end{array}$

Low SES (bottom quintile of IMD score) $\quad 18 / 48(37.5 \%) \quad 10 / 20(50.0 \% \quad 8 / 28(28.6 \%) \quad 0.3$

$\begin{array}{llll}\text { High blood pressure } & & & 10 / 29 \\ \text { High lipids } & 16 / 48(33.3 \%) & 7 / 21(33.3 \%) & (34.4 \%) \\ \text { Type 2 diabetes } & 17 / 43(39.5 \%) & 4 / 16(25.0 \%) & 6 / 27(22.2 \%) \\ \text { Polycystic ovarian syndrome } & 5 / 50(10.0 \%) & 2 / 20(10.0 \%) & 3 / 29(10.3 \%) \\ \text { Sleep apnoea } & \mathrm{n} / \mathrm{a} & \mathrm{n} / \mathrm{a} & 5 / 18(27.8 \%) \\ \text { NAFLD } & \mathrm{n} / \mathrm{a} & \mathrm{n} / \mathrm{a} & 4 / 29(13.7 \%) \\ & \mathrm{n} / \mathrm{a} & \mathrm{n} / \mathrm{a} & 3 / 29(10.3 \%)\end{array}$

Table 1 - demographics and baseline co-morbidity data for complete cohort, those undergoing surgery, and those not undergoing surgery. NAFLD $=$ non-alcoholic fatty liver disease, $\mathrm{n} / \mathrm{a}=$ not applicable (those not undergoing surgery did not have full evaluation). SES = socio-economic status. For cardio-metabolic screening, denominator states total number tested and numerator number testing positive. 